\title{
On the Influence of the Bridge on Triplet State Delocalization in Linear Porphyrin Oligomers
}

\author{
Sabine Richert, ${ }^{*}{ }^{\dagger}$ Bart Limburg, ${ }^{\ddagger}$ Harry L. Anderson, ${ }^{\ddagger \odot}$ and Christiane R. Timmel ${ }^{*}{ }^{\dagger}$ (i) \\ ${ }^{\dagger}$ Centre for Advanced Electron Spin Resonance (CAESR), University of Oxford, South Parks Road, Oxford OX1 3QR, United \\ Kingdom \\ ${ }^{*}$ Chemistry Research Laboratory, Department of Chemistry, University of Oxford, 12 Mansfield Road, Oxford OX1 3TA, United \\ Kingdom
}

\section{Supporting Information}

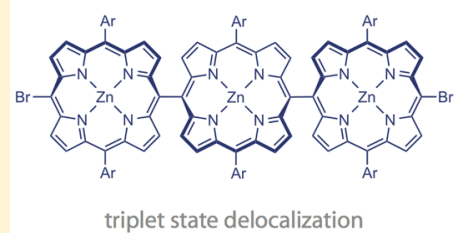

triplet state delocalization

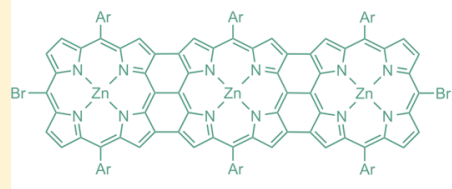

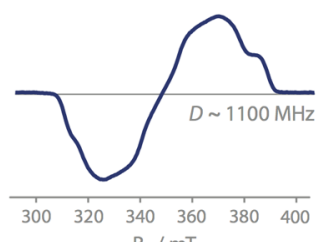

$\mathrm{B}_{0} / \mathrm{mT}$

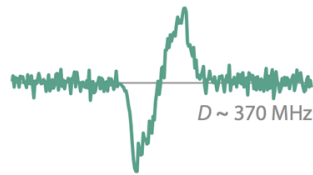

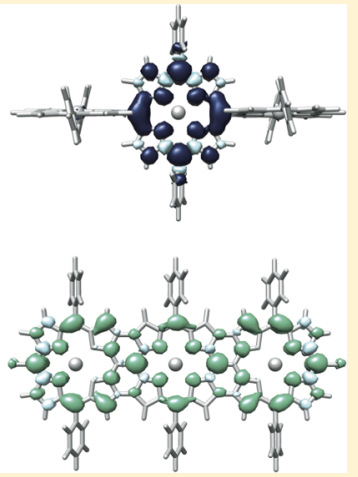

ABSTRACT: The extent of triplet state delocalization is investigated in rigid linear zinc porphyrin oligomers as a function of interporphyrin bonding characteristics, specifically in meso-meso singly linked and $\beta$, meso, $\beta$ fused structures, using electron paramagnetic resonance techniques. The results are compared with those of earlier measurements on porphyrin oligomers with alkyne linkers exhibiting different preferred conformations. It is shown that dihedral angles near $90^{\circ}$ between the porphyrin planes in directly meso-to-meso linked porphyrin oligomers lead to localization of the photoexcited triplet state on a single porphyrin unit, whereas previous work demonstrated even delocalization over two units in meso-to-meso ethyne or butadiynebridged oligomers, where the preferred dihedral angles amount to roughly $30^{\circ}$ and $0^{\circ}$, respectively. The triplet states of fused porphyrin oligomers (i.e., porphyrin tapes) exhibit extended conjugation and even delocalization over more than two porphyrin macrocycles, in contrast to meso-to-meso ethyne or butadiyne-bridged oligomers, where the spin density distribution in molecules composed of more than two porphyrin units is not evenly spread across the oligomer chain.

\section{INTRODUCTION}

Triplet excitons are important intermediates in many processes of biological or technological relevance. ${ }^{1-5}$ The kinetics of their formation and their properties have a major influence on the performance of devices relying on singlet or triplet exciton fission or fusion, such as organic semiconductors. ${ }^{6,7}$ Triplet formation is a common loss mechanism in organic solar cells. ${ }^{8-10}$ Furthermore, the extent of delocalization of the photoexcited triplet state has recently been shown to impact the performance of organic photovoltaic devices and organic light emitting diodes. ${ }^{11,12}$

A thorough understanding of the factors influencing triplet state delocalization could advance the development of structure-property relations guiding the design of new functional materials. A targeted modification of the triplet state properties should allow control over the loss mechanisms and therefore enable enhanced device performance.

In this study, the influence of the linker connecting the individual units and the structural rigidity of the oligomeric chain on triplet state delocalization is explored in linear singly linked and fused porphyrin assemblies. The type of the linker determines the preferred dihedral angle between the porphyrin planes, which approaches $90^{\circ}$ as the linker is shortened from a butadiyne group to a direct meso-to-meso bond. ${ }^{13,14}$ At the same time, the increased steric hindrance upon shortening of the linker renders the oligomer chain more rigid and reduces the conformational spread.

Exploiting the paramagnetic nature of triplet states, electron paramagnetic resonance (EPR) spectroscopy has been shown to be particularly suitable for the study of triplet delocalization in porphyrin ${ }^{15-19}$ and other ${ }^{20}$ oligomers. If the orientation of the zero-field splitting (ZFS) tensor is known, information about delocalization in a series of oligomers can be obtained from changes in the ZFS $D$-value by comparison with a suitable monomer spectrum. The cube of the average electron-electron

Received: June 22, 2017

Published: August 15, 2017 

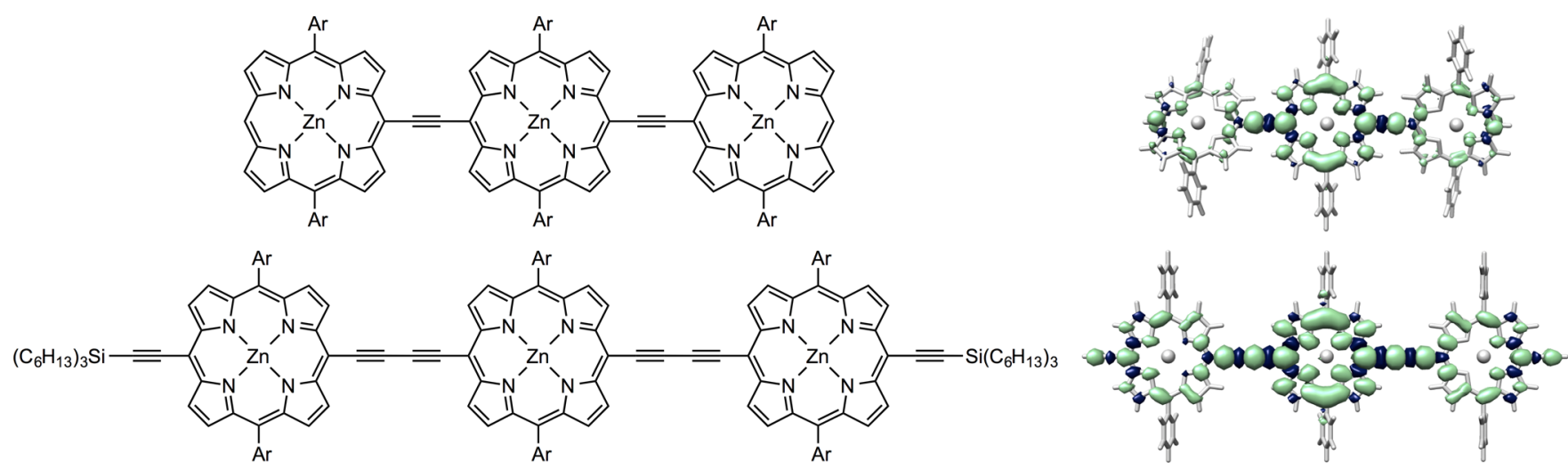

Figure 1. Structures of previously investigated ${ }^{18,22}$ linear ethyne and butadiyne-bridged zinc porphyrin trimers (left) and corresponding spin density distributions calculated by DFT (right). The spin density is unevenly distributed with the majority of the spin density on the central porphyrin unit. "Ar" represents a solubilizing aryl side group, 3,5-bis(2-(2-(2-methoxyethoxy)ethoxy)ethoxy)phenyl (top) or 3,5-bis(trihexylsilyl)phenyl (bottom).

distance, $r$, is inversely proportional to the $D$-value, related to the width of the transient EPR triplet spectrum ${ }^{19}$

$$
D=\frac{3}{4} \frac{\mu_{0}}{4 \pi h}\left(g_{\mathrm{e}} \beta_{\mathrm{e}}\right)^{2}\left\langle\frac{1-3 \cos ^{2} \theta}{r^{3}}\right\rangle
$$

where $\theta$ refers to the angle between the dipolar $Z$-axis and the spin-spin vector, $g_{\mathrm{e}}$ is the electronic $g$-factor, $\beta_{\mathrm{e}}$ is the Bohr magneton, $\mu_{0}$ is the vacuum permeability, and the angular brackets indicate the expectation value. The angle $\theta$ characterizes the shape of the spin density distribution and is equal to $90^{\circ}$ in the case of an oblate spin density distribution and equal to $0^{\circ}$ in the case of a prolate one. As long as the spin distribution stays invariant and there are no other significant contributions to the ZFS tensor apart from the spin-spin contribution, increased delocalization should manifest itself in a reduction of the width of the triplet EPR spectrum, since the width of the spectrum is equal to $2|D|$.

Further information on triplet state delocalization can be obtained from triplet state electron nuclear double resonance (ENDOR) measurements, especially in cases where the orientation of the ZFS tensor is likely to be different for individual molecules within a series. ${ }^{18,21,22}$ The magnitudes of the measured hyperfine coupling constants are directly proportional to the triplet spin density. Therefore, if the triplet spin density is equally distributed in an oligomer with $N$ identical units, the hyperfine coupling constants of the oligomer should be reduced by a factor of $1 / N$ with respect to those of the monomer.

Previous EPR studies on triplet state delocalization in linear ethyne and butadiyne-bridged zinc porphyrin oligomers have shown that even delocalization of the triplet state is limited to about two porphyrins units. ${ }^{18,22}$ For even delocalization to be observed, these two porphyrin units additionally need to be equivalent. $^{22}$ These experimental results from EPR and ENDOR are in excellent qualitative agreement with density functional theory (DFT) calculations (B3LYP/EPRII). For the longer oligomers, the calculations reveal that the majority of the spin density is located on the central porphyrin units of the structures, as illustrated in Figure 1 for the example of meso-tomeso ethyne and butadiyne bridged linear zinc porphyrin trimers. The corresponding ZFS and hyperfine coupling parameters are however in agreement with a description of Frenkel-type exciton delocalization (i.e., delocalization of the position of the center of mass of the electron and hole of a strongly bound electron-hole pair). ${ }^{20,23}$

DFT calculations furthermore predict that the preferred dihedral angle between two adjacent porphyrin units amounts to approximately $0^{\circ}$ in butadiyne-bridged and roughly $30^{\circ}$ in ethyne-bridged oligomers. While the barrier to rotation of the porphyrin units about the linker is comparatively low in butadiyne-bridged oligomers leading to a broad distribution of conformations, ${ }^{24}$ this conformational distribution is expected to be narrower for ethyne-bridged oligomers due to a higher rotational barrier resulting from increased steric constraints and increased $\pi$-conjugation. ${ }^{25,26}$

Results from excitation wavelength dependent EPR measurements on butadiyne-bridged porphyrins ${ }^{27}$ suggest that the excited triplet state localizes on a single porphyrin unit if conformations with interporphyrin dihedral angles near $90^{\circ}$ are predominantly probed. However, since the fraction of excited molecules with angles close to $90^{\circ}$ was low in these measurements and the corresponding changes in signal intensity fairly small, only tentative conclusions could be drawn.

Here we use transient EPR and ENDOR techniques to investigate triplet state delocalization in a series of linear zinc porphyrin oligomers, directly linked in meso position as shown in Figure 2. Monomer, dimer, and trimer will be referred to as $\mathbf{P} \mathbf{1}_{\mathrm{H}}, \mathbf{P} \mathbf{2}_{\mathrm{H}}$, and $\mathbf{P} \mathbf{3}_{\mathrm{H}}$, respectively. In these oligomers, the interporphyrin dihedral angles have previously been shown to amount to roughly $90^{\circ}$ with very little conformational flexibility. ${ }^{28-30}$ The results obtained for the singly linked oligomers will be contrasted with those obtained for their fused counterparts, where two additional covalent bonds in $\beta$ position introduce additional $\pi$-conjugated connections and force the individual porphyrin macrocycles into a planar, tapelike structure.

\section{RESULTS AND DISCUSSION}

The transient cW EPR spectra of $\mathbf{P} \mathbf{1}_{\mathrm{H}}, \mathbf{P} \mathbf{2}_{\mathrm{H}}$, and $\mathbf{P} \mathbf{3}_{\mathrm{H}}$ are shown in Figure 3 (left). All EPR data were acquired at X-band (9.75 $\mathrm{GHz}$ ) in frozen 2-methyltetrahydrofuran (MTHF) solution at $20 \mathrm{~K}$. Unless otherwise stated, the samples were excited at 532 $\mathrm{nm}(\sim 2 \mathrm{~mJ})$, using the second harmonic of a Nd:YAG laser operated at $20 \mathrm{~Hz}$. Further details on the sample preparation and experimental setup for the EPR measurements are given in the Supporting Information (SI).

Since the EPR spectra were recorded using direct detection, a positive signal corresponds to an absorptive (a) transition, 


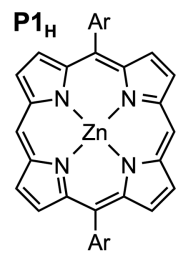

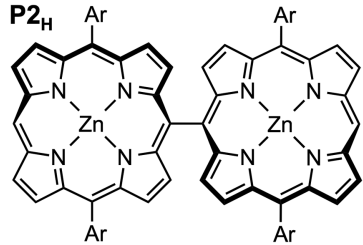

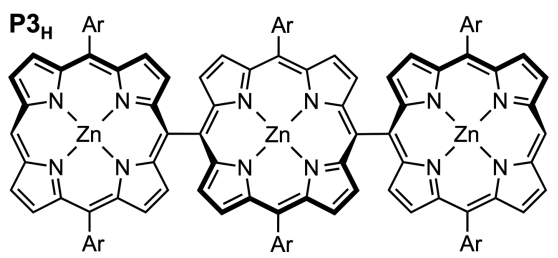

Figure 2. Chemical structures of the investigated linear zinc porphyrin oligomers $\mathbf{P} \mathbf{1}_{\mathrm{H}}, \mathbf{P} \mathbf{2}_{\mathrm{H}}$, and $\mathbf{P} \mathbf{3}_{\mathrm{H}}$. The porphyrin side group "Ar" represents an aryl substituent, 3,5-bis(trihexylsilyl)phenyl, which provides high solubility and prevents aggregation. Further details on the synthesis and characterization of the compounds are given in the SI.

whereas negative signals result from emissive (e) transitions. The observed spin polarization in $\mathbf{P} \mathbf{1}_{\mathrm{H}}$, aaaeee, is consistent with results obtained for similar zinc porphyrin monomers and indicates a selective population of the out-of-plane triplet sublevel $(Z)$ by intersystem crossing. A positive $D$-value is assumed, as confirmed for zinc porphyrin monomers with terminal ethyne groups in the meso-positions by magnetophotoselection experiments and DFT calculations. ${ }^{21}$

The comparison of the triplet state EPR spectra of the singly linked oligomers (Figure 3, left) shows that no significant changes in the ZFS parameter $D$ or the relative triplet sublevel populations occur within the series of molecules since the spectral width and the overall spectral shape remain almost invariant. The ZFS parameters and relative triplet state sublevel populations obtained from a numerical simulation of the spectra are shown in the SI (Figure S4). The similarity of the triplet spectra of $\mathbf{P} \mathbf{1}_{\mathrm{H}}, \mathbf{P} \mathbf{2}_{\mathrm{H}}$, and $\mathbf{P} \mathbf{3}_{\mathrm{H}}$ might indicate that the delocalization of the triplet excited state is similar in all of the investigated singly linked compounds and does therefore not exceed a single porphyrin unit. However, since the shape of the spin-density distribution in the longer oligomers is a priori not known, a confirmation of this tentative interpretation by pulse ENDOR spectroscopy, if attainable (fast relaxation and/or short triplet state lifetimes are limiting factors, see below), is desirable.

The canonical orientations in the spectra are assigned to the three ZFS tensor axes in Figure 3 under the assumption of a positive $D$-value. At the field positions corresponding to these three orientations, proton Mims ENDOR spectra were taken and are shown in the SI for reference. To interpret the orientation selective ENDOR spectra and assign the couplings to the individual protons of the structure, DFT calculations of the hyperfine coupling tensors were performed using ORCA (B3LYP/EPRII). ${ }^{31}$ Technical details on these calculations are provided in the SI. A visualization of the calculated proton and nitrogen hyperfine tensors for $\mathbf{P} \mathbf{1}_{\mathbf{H}}$ is shown in Figure 3 (center and right) together with an assignment of the ZFS tensor axes to the molecular axes. The proton hyperfine tensors are shown in green and their relative size reflects the magnitude of the couplings. The couplings measured using ENDOR should correspond to a projection of the hyperfine tensors onto the indicated ZFS tensor axes. In our assignment, the $Y$ axis corresponds to the axis along the aryl groups, whereas $Z$ is assigned to the out-of-plane axis in analogy to similar porphyrins. $^{21}$ A pronounced proton coupling with a magnitude of roughly $-4 \mathrm{MHz}$ is observed in the ENDOR spectra recorded at the $X$ - and $Z$-orientations (cf. SI, Figure S7, right) and corresponds to the proton labeled $\mathrm{H}_{1}$ in Figure 3.

To confirm the extent of triplet state delocalization in the longer oligomers, the ENDOR spectra recorded at the $Z^{-}$ orientation were compared. The detection of the ENDOR couplings at the outmost position of the triplet spectrum $(Z)$ has the advantage that no spurious contributions from other orientations need to be considered. Furthermore, the comparatively large coupling of $\sim-4 \mathrm{MHz}$ resulting from $\mathrm{H}_{1}$ is quite pronounced at this orientation and should therefore be easily comparable between the oligomers. An overlay of the corresponding spectra for $\mathbf{P} \mathbf{1}_{\mathbf{H}}, \mathbf{P} \mathbf{2}_{\mathrm{H}}$, and $\mathbf{P} \mathbf{3}_{\mathbf{H}}$ is shown in Figure 4 (left). No significant change in the magnitude of the
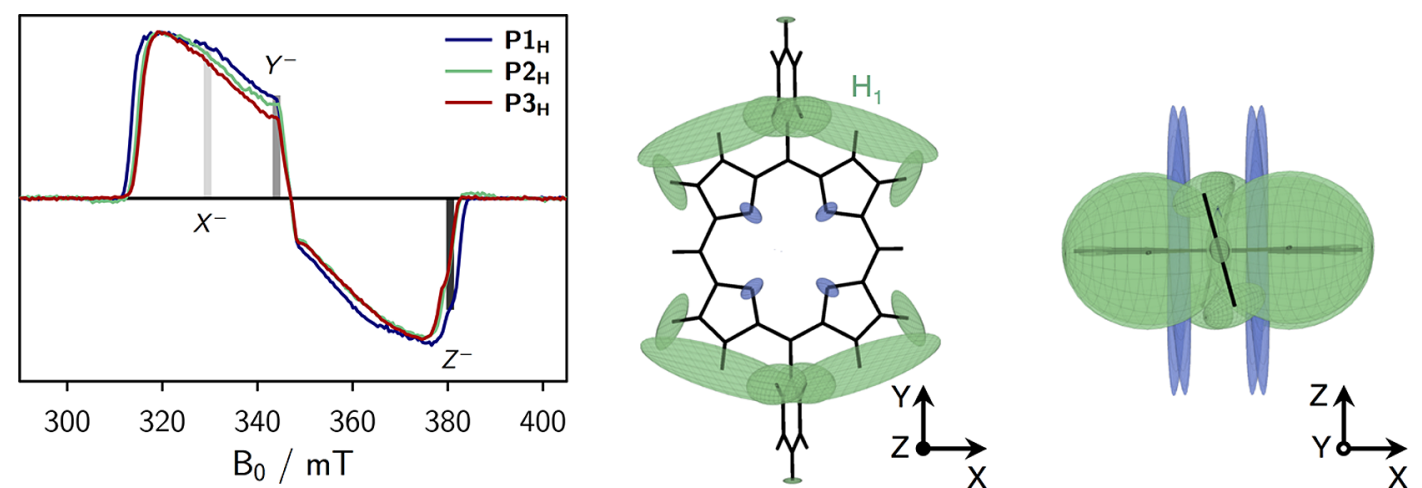

Figure 3. Overlay of the transient cw EPR spectra of the singly linked compounds (left), and visualization of the calculated hyperfine coupling tensors and definition of the ZFS tensor axes in top view (center) and side view (right). Proton hyperfine tensors are shown in green, whereas nitrogen hyperfine tensors are depicted in blue. The field positions corresponding to the $X^{-}, Y^{-}$and $Z^{-}$orientations are indicated in the spectra. A large hyperfine coupling exceeding $-10 \mathrm{MHz}$ is calculated for the proton in meso-position of the porphyrin core. This large coupling is omitted in the figure for clarity. The EPR data were acquired in frozen MTHF at $20 \mathrm{~K}$. 

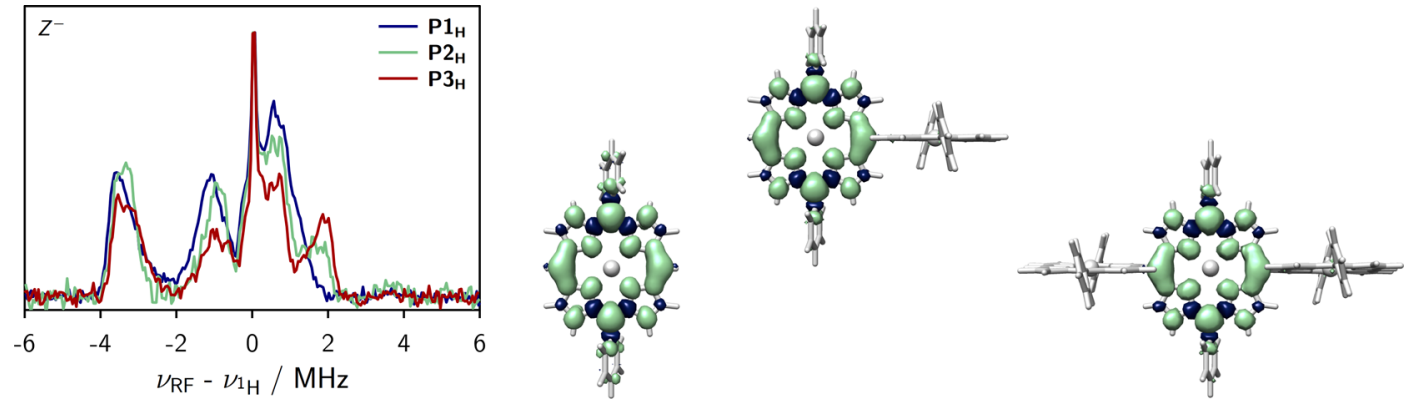

Figure 4. Comparison of the proton Mims ENDOR spectra of $\mathbf{P} \mathbf{1}_{\mathrm{H}}, \mathbf{P} \mathbf{2}_{\mathrm{H}}$, and $\mathbf{P} \mathbf{3}_{\mathrm{H}}$ recorded at magnetic field positions corresponding to the $Z^{-}$ transition and visualization of the spin density distributions in $\mathbf{P} \mathbf{1}_{\mathbf{H}}, \mathbf{P} \mathbf{2}_{\mathrm{H}}$, and $\mathbf{P} \mathbf{3}_{\mathrm{H}}$ calculated by DFT. The ENDOR spectra were recorded at $20 \mathrm{~K}$ and pulse lengths of $16 \mathrm{~ns}$ were applied. To compensate for blind spots, three ENDOR spectra with $\tau$ values of 120, 180, and 240 ns were averaged in all cases. Although calculations predict the spin density to localize on the central unit in $\mathbf{P} \mathbf{3}_{\mathbf{H}}$ as shown in the figure, solvent effects or changes of the geometry in solution might lead to localization of the triplet state on any one of the three units.
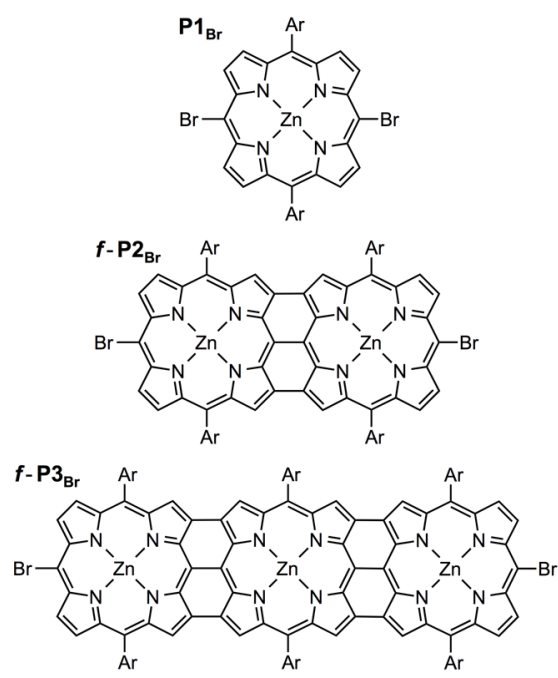

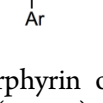
Figure 5. Chemical structures of the fused porphyrin oligomers $\mathbf{P} \mathbf{1}_{\mathrm{Br}}, \boldsymbol{f}$-P2 $\mathbf{2}_{\mathrm{Br}}$, and $f$-P $\mathbf{3}_{\mathrm{Br}}$ (left), corresponding transient $\mathrm{cw}$ EPR spectra and simulations including sim
bis(trihexylsilyl)phenyl.

hyperfine coupling of $\sim-4 \mathrm{MHz}$ is observed, confirming that the excited triplet state remains localized on a single porphyrin unit in these singly linked oligomers where the dihedral angle between adjacent porphyrin planes is close to $90^{\circ}$. DFT calculations of the spin density distribution further confirm that the triplet state localizes only on one of the porphyrin units in the dimer and trimer, as shown in Figure 4 (right). ${ }^{32}$

Upon oxidation, the meso-meso singly linked porphyrin oligomers can be transformed into triply linked porphyrin tapes. $^{28}$ The structures are shown in Figure 5 (left), and the synthesis and characterization are presented in the SI. Compared to the singly linked oligomers, two additional covalent $\beta-\beta$ bonds are formed between the porphyrin macrocycles, introducing additional $\pi$-conjugated connections and forcing the porphyrin $\pi$-systems into a coplanar conformation with maximum $\pi$-overlap.

The triplet yield of the fused porphyrin dimer with terminal protons in meso-positions has previously been shown to be negligible. ${ }^{33}$ To increase the triplet yield for the measurements in this study, the series of fused porphyrin oligomers was therefore prepared with $-\mathrm{Br}$ substituents in the meso-positions. The structures from monomer to trimer are referred to as $\mathbf{P} \mathbf{1}_{\mathrm{Br}}$, $f-\mathbf{P} 2_{\mathrm{Br}}$, and $f-\mathbf{P} 3_{\mathrm{Br}}$. The triplet yield for $f-\mathbf{P} 2_{\mathrm{Br}}$ (with different aryl side chains) has previously been estimated to amount to roughly 0.12 by analysis of transient absorption spectra. ${ }^{33}$ Due to the presence of the terminal $-\mathrm{Br}$ substituents, the porphyrin in-plane triplet sublevels $(X, Y)$ are primarily populated by intersystem crossing, which results in the eeeaaa polarization pattern observed in the triplet state cw EPR spectra of these compounds (cf. Figure 5, center).

While the triplet yield, spin relaxation properties, and triplet lifetime of $\mathbf{P} \mathbf{1}_{\mathrm{Br}}$ are similar to those of a typical porphyrin monomer, the transient cw EPR experiments on the fused porphyrins resulted in very low signal intensities due to a very short triplet state lifetime ${ }^{33}$ and possibly also fast spin relaxation. For these reasons, no pulse EPR or ENDOR experiments could be carried out on $f-\mathbf{P} 2_{\mathrm{Br}}$ and $f-\mathbf{P} 3_{\mathrm{Br}}$. Proton ENDOR experiments have however been performed on the singly linked oligomers with terminal $-\mathrm{Br}$ groups and are shown in the SI for reference. Compared to their counterparts with terminal protons, the hyperfine spectra of these compounds are very similar and show the same trends.

For the series of fused porphyrin oligomers, information about delocalization could thus only be obtained by evaluation of the changes in the ZFS parameter $D$. Since previous studies have shown that precise information about the orientation of the ZFS tensor is required in order to correctly interpret any changes in the spectral width of the EPR spectra, ${ }^{21,22}$ DFT 
calculations were performed to support the experimentally observed trends. The latter predict positive $D$-values for all investigated fused porphyrin arrays, suggesting no marked changes of the orientation of the ZFS tensor and thus the factor $\left(1-3 \cos ^{2} \theta\right)$ in eq 1 . If the orientation of the ZFS tensor remains invariant, delocalization of the excited triplet state beyond a single unit should manifest itself in a reduction of the width of the triplet EPR spectrum.

The transient $\mathrm{cw}$ EPR spectra of $\boldsymbol{f}-\mathbf{P} \mathbf{2}_{\mathrm{Br}}$, and $\boldsymbol{f}-\mathbf{P} \mathbf{3}_{\mathrm{Br}}$, obtained when exciting the molecules at their respective absorption maxima of 565 and $670 \mathrm{~nm}$ using an OPO (optical parametric oscillator) system $(2 \mathrm{~mJ}, 20 \mathrm{~Hz}, 5 \mathrm{~ns}$ pulse length), are shown in Figure 5 (center). To quantify the ZFS parameters as well as the relative initial populations of the triplet sublevels, numerical simulations were carried out using the MATLAB software package EasySpin ${ }^{34}$ in combination with a home-written MATLAB fitting routine. The triplet state parameters, $D$ and $E$, as well as the relative populations of the triplet sublevels, obtained as a result from the fit, are indicated in the figure. ${ }^{35}$

A significant reduction in $\mathrm{D}$ from $1200 \mathrm{MHz}$ to about 590 $\mathrm{MHz}$ is observed experimentally between $\mathbf{P} \mathbf{1}_{\mathrm{Br}}$ and $f-\mathbf{P} \mathbf{2}_{\mathrm{Br}}$, suggesting increased delocalization of the triplet state wavefunction. When the $\pi$-system is further extended in $f$-P $3_{\mathrm{Br}}$, the $D$-value is decreased even further to about $370 \mathrm{MHz}$.

To evaluate whether the decreased $D$-value is consistent with an even delocalization of the triplet wavefunction, the experimentally observed changes in $D$ were contrasted with the calculated values obtained from DFT. Only relative changes shall be compared here, since it is known that DFT calculations generally underestimate the ZFS parameters. ${ }^{36}$ Comparisons of parameter trends, however, have been shown to be reliable in previous studies on similar systems. ${ }^{18,22,27}$

The relative $D$-values in the series $\mathbf{P} \mathbf{1}_{\mathrm{Br}}: f-\mathbf{P} \mathbf{2}_{\mathrm{Br}}: f-\mathbf{P} \mathbf{3}_{\mathrm{Br}}$, as calculated by DFT, amount to $1: 0.58: 0.33$ and correspond to the triplet spin density distributions shown in Figure 5 (right). The absolute values obtained from DFT are listed in the SI for reference. These calculated changes are in excellent agreement with the experimental ratio of 1:0.49:0.31, indicating even delocalization of the triplet state in fused porphyrin arrays as illustrated in Figure 5.

In contrast to meso-to-meso ethyne or butadiyne-bridged porphyrins where uneven delocalization of the triplet state was observed in oligomers with more than two porphyrin units, this study showed that even delocalization can be achieved in a fused, fully conjugated, porphyrin trimer. The observation of even delocalization of the triplet spin density in tapelike porphyrin molecules relates well to the extraordinary properties of their singlet excited states such as absorption spectra reaching far into the near-infrared. ${ }^{28}$

\section{CONCLUSIONS}

In this study, the influence of different bridges on triplet state delocalization in linear zinc porphyrin oligomers was explored by EPR spectroscopy. The results obtained on porphyrins linked by a direct meso-to-meso bond are consistent with previous studies on ethyne and butadiyne-bridged oligomers $^{18,21,22,27}$ and clearly indicate that the triplet state localizes on a single porphyrin unit as the dihedral angle between adjacent porphyrin planes approaches $90^{\circ}$. No significant differences in the hyperfine coupling constants measured by triplet state ENDOR are observed between $\mathbf{P} \mathbf{1}_{\mathbf{H}}$ and the longer oligomers $\mathbf{P} \mathbf{2}_{\mathbf{H}}$ and $\mathbf{P} \mathbf{3}_{\mathbf{H}}$, showing that the extent of the triplet state wavefunction is approximately identical within this series. The experimental results are fully consistent with DFT calculations, suggesting that the triplet state localizes on only one of the porphyrin units in $\mathbf{P} 3_{\mathrm{H}}$.

In linear porphyrin tapes, where the porphyrin units are forced to adopt a coplanar conformation by two additional covalent bonds between the porphyrin macrocycles, even delocalization of the triplet state was experimentally demonstrated by analysis of the zero-field splitting parameters. Positive $D$-values were obtained from DFT calculations for all molecules of the series $\mathrm{P} \mathbf{1}_{\mathrm{Br}}, \boldsymbol{f}-\mathrm{P} \mathbf{2}_{\mathrm{Br}}$, and $\boldsymbol{f}-\mathrm{P} \mathbf{3}_{\mathrm{Br}}$. With respect to the values obtained for the monomer, $\mathbf{P} \mathbf{1}_{\mathrm{Br}}$, DFT predicts a reduction in $D$ by about $67 \%$ for $f-P 3_{\mathrm{Br}}$. This is in excellent agreement with the results from numerical simulations of the experimental spectra, where a reduction by $69 \%$ was observed, and indicates even delocalization of the triplet state wavefunction over the entire molecule.

The even delocalization of the excited triplet state in fused porphyrin oligomers was contrasted with the observed uneven delocalization in ethyne or butadiyne-bridged oligomers with more than two porphyrin units. Based on their singlet and triplet state properties, the investigated linear, fully conjugated, porphyrin tapes seem to be ideal candidates for molecular wires. ${ }^{37}$ However, the investigation of triplet state delocalization in these molecules is limited by small triplet yields and short triplet state lifetimes.

\section{ASSOCIATED CONTENT}

\section{S Supporting Information}

The Supporting Information is available free of charge on the ACS Publications website at DOI: 10.1021 /jacs.7b06518.

Synthetic procedures and characterization of the compounds, additional experimental data, and details of the DFT calculations (PDF)

\section{AUTHOR INFORMATION}

\section{Corresponding Authors}

*sabine.richert@chem.ox.ac.uk

*christiane.timmel@chem.ox.ac.uk

ORCID

Sabine Richert: 0000-0002-2990-974X

Harry L. Anderson: 0000-0002-1801-8132

Christiane R. Timmel: 0000-0003-1828-7700

Notes

The authors declare no competing financial interest.

\section{ACKNOWLEDGMENTS}

Financial support from the EPSRC (EPL011972/1 and EP/ N017188/1) and the ERC (Grant 320969) is gratefully acknowledged. The authors would like to acknowledge the use of the University of Oxford Advanced Research Computing (ARC) facility in carrying out this work (http://dx.doi.org/10. 5281/zenodo.22558). We thank the EPSRC UK National Mass Spectrometry Facility at Swansea University for MALDI spectra. We are grateful to Prof. William Barford for fruitful discussions.

\section{REFERENCES}

(1) Levanon, H.; Norris, J. R. Chem. Rev. 1978, 78, 185-198.

(2) Budil, D. E.; Thurnauer, M. C. Biochim. Biophys. Acta, Bioenerg. 1991, 1057, 1-41.

(3) Lubitz, W.; Lendzian, F.; Bittl, R. Acc. Chem. Res. 2002, 35, 313320. 
(4) Wohlgenannt, M.; Jiang, X. M.; Vardeny, Z. V.; Janssen, R. A. J. Phys. Rev. Lett. 2002, 88, 197401.

(5) Singh-Rachford, T. N.; Castellano, F. N. Coord. Chem. Rev. 2010, 254, 2560-2573.

(6) Köhler, A.; Bässler, H. J. Mater. Chem. 2011, 21, 4003-4011.

(7) Walker, B. J.; Musser, A. J.; Beljonne, D.; Friend, R. H. Nat. Chem. 2013, 5, 1019-1024.

(8) Veldman, D.; Offermans, T.; Sweelssen, J.; Koetse, M. M.; Meskers, S. C. J.; Janssen, R. A. J. Thin Solid Films 2006, 511-512, 333-337.

(9) Chang, W.; Congreve, D. N.; Hontz, E.; Bahlke, M. E.; McMahon, D. P.; Reineke, S.; Wu, T. C.; Bulović, V.; van Voorhis, T.; Baldo, M. A. Nat. Commun. 2015, 6, 6415.

(10) Kraus, H.; Heiber, M. C.; Väth, S.; Kern, J.; Deibel, C.; Sperlich, A.; Dyakonov, V. Sci. Rep. 2016, 6, 29158.

(11) Bakulin, A. A.; Rao, A.; Pavelyev, V. G.; van Loosdrecht, P. H. M.; Pshenichnikov, M. S.; Niedzialek, D.; Cornil, J.; Beljonne, D.; Friend, R. H. Science 2012, 335, 1340-1344.

(12) Rao, A.; Chow, P. C. Y.; Gélinas, S.; Schlenker, C. W.; Li, C.-Z.; Yip, H.-L.; Jen, A. K.-Y.; Ginger, D. S.; Friend, R. H. Nature 2013, 500, 435-440.

(13) Yoshida, N.; Ishizuka, T.; Osuka, A.; Jeong, D. H.; Cho, H. S.; Kim, D.; Matsuzaki, Y.; Nogami, A.; Tanaka, K. Chem. - Eur. J. 2003, 9, $58-75$.

(14) Khoury, R. G.; Jaquinod, L.; Smith, K. M. Chem. Commun. 1997, 1057-1058.

(15) Angiolillo, P. J.; Lin, V. S.-Y.; Vanderkooi, J. M.; Therien, M. J. J. Am. Chem. Soc. 1995, 117, 12514-12527.

(16) Di Valentin, M.; Kay, C. W. M.; Giacometti, G.; Möbius, K. Chem. Phys. Lett. 1996, 248, 434-441.

(17) Lendzian, F.; Bittl, R.; Telfer, A.; Lubitz, W. Biochim. Biophys. Acta, Bioenerg. 2003, 1605, 35-46.

(18) Tait, C. E.; Neuhaus, P.; Peeks, M. D.; Anderson, H. L.; Timmel, C. R. J. Am. Chem. Soc. 2015, 137, 8284-8293.

(19) Richert, S.; Tait, C. E.; Timmel, C. R. J. Magn. Reson. 2017, 280, $103-116$.

(20) Hintze, C.; Korf, P.; Degen, F.; Schütze, F.; Mecking, S.; Steiner, U. E.; Drescher, M. J. Phys. Chem. Lett. 2017, 8, 690-695.

(21) Tait, C. E.; Neuhaus, P.; Anderson, H. L.; Timmel, C. R. J. Am. Chem. Soc. 2015, 137, 6670-6679.

(22) Richert, S.; Bullard, G.; Rawson, J.; Angiolillo, P. J.; Therien, M. J.; Timmel, C. R. J. Am. Chem. Soc. 2017, 139, 5301-5304.

(23) Barford, W. J. Phys. Chem. A 2013, 117, 2665-2671.

(24) Peeks, M. D.; Neuhaus, P.; Anderson, H. L. Phys. Chem. Chem. Phys. 2016, 18, 5264-5274.

(25) Lin, V. S.-Y.; Therien, M. J. Chem. - Eur. J. 1995, 1, 645-651.

(26) Rintoul, L.; Harper, S. R.; Arnold, D. P. Phys. Chem. Chem. Phys. 2013, 15, 18951-18964.

(27) Tait, C. E.; Neuhaus, P.; Peeks, M. D.; Anderson, H. L.; Timmel, C. R. Phys. Chem. Chem. Phys. 2016, 18, 5275-5280.

(28) Tsuda, A.; Osuka, A. Science 2001, 293, 79-82.

(29) Tsuda, A.; Furuta, H.; Osuka, A. J. Am. Chem. Soc. 2001, 123, 10304-10321.

(30) Kim, D.; Osuka, A. J. Phys. Chem. A 2003, 107, 8791-8816.

(31) Neese, F. Wiley Interdiscip. Rev.: Comput. Mol. Sci. 2012, 2, 7378.

(32) ENDOR spectra at the $X^{-}$and $Y^{-}$orientations were also recorded for $\mathbf{P} \mathbf{1}_{\mathrm{H}}, \mathbf{P} \mathbf{2}_{\mathrm{H}}$, and $\mathbf{P} \mathbf{3}_{\mathrm{H}}$. They support the conclusions obtained by comparison of the spectra in $Z^{-}$orientation and are presented in the SI for reference.

(33) McEwan, K. J.; Fleitz, P. A.; Rogers, J. E.; Slagle, J. E.; McLean, D. G.; Akdas, H.; Katterle, M.; Blake, I. M.; Anderson, H. L. Adv. Mater. 2004, 16, 1933-1935.

(34) Stoll, S.; Schweiger, A. J. Magn. Reson. 2006, 178, 42-55.

(35) Significant differences in linewidth are noted between some of the transient EPR triplet spectra (see, for instance, $\mathbf{P} \mathbf{1}_{\mathrm{Br}}$ vs $\mathbf{P} \mathbf{1}_{\mathrm{H}}$ or $f$ $\mathbf{P 2} \mathbf{B r}_{\mathrm{Br}}$ ). The origin of this is presently not clear, albeit fully reproducible. Changes in end group, oligomer length, etc. will affect the linewidth via factors such as altered contributions of unresolved hyperfine couplings, relaxation, small changes in conformation, and consequently different strains in $g, D$, and $E$. A physically meaningful deconvolution of these effects is presently not possible.

(36) Neese, F. J. Chem. Phys. 2007, 127, 164112.

(37) Sedghi, G.; Esdaile, L. J.; Anderson, H. L.; Martin, S.; Bethell, D.; Higgins, S. J.; Nichols, R. J. Adv. Mater. 2012, 24, 653-657. 